Daniel Allenbach

\title{
Eine >heroische< Neunte? Dmitri Schostakowitschs Neunte Sinfonie im Vergleich mit Ludwig van Beethovens Sinfonien Nr. 3 und ${ }^{1}$
}

Wenn es im folgenden Beitrag um eine Spurensuche nach Einflüssen von Ludwig van Beethovens Sinfonien Nr. 3 und 9 auf Dmitri Schostakowitsch Neunte gehen soll, geschieht dies im vollen Bewusstsein, dass solche Parallelbefunde und potentielle Bezüge vielfach auf schwankendem Grund stehen. Schließlich handelt es sich bei Schostakowitschs Opus ebenso wie bei Beethovens Werken um eine eigenständige und für sich sprechende Schöpfung und keineswegs um eine Kopie um der Kopie willen, quasi eine reine Fingerübung. Wo deshalb etwa ein Akkord, ein Rhythmus, eine melodische Linie an die Vorlage anklingen sollen und wo sich ihre Verwendung aus werkimmanenter schöpferischer Notwendigkeit ergibt, ist durchaus Interpretationssache. Angesichts der Konstanz solch auffälliger Bezüge über das ganze Werk und im Hinblick auf ihre politischen Implikationen im Umfeld der Entstehung der Sinfonie sei der Versuch aber dennoch gewagt.

Dass Beethovens Sinfonien und ganz besonders seine Neunte die nachfolgenden Komponistengenerationen beeinflusst haben, ist gleichsam ein Gemeinplatz, der anhand zahlreicher Beispiele belegt werden kann. Von Ernst Theodor Amadeus Hoffmanns Rezension der Fünften, in der dieser das durch Beethovens Musik eröffnete »Reich des Ungeheuren und Unermesslichen « schildert, ${ }^{2}$ über Johannes Brahms, der sich mit seinen eigenen Sinfonien schwertut, da er »immer so einen Riesen (Beethoven) hinter sich marschieren hört«,3 und Richard Wagner, der insbesondere in der Neunten den zentralen Vorläufer seines »Kunstwerks der Zukunft« sieht, ${ }^{4}$ scheint bis heute immer

1 Dieser Beitrag basiert auf einer früheren, unpublizierten Arbeit des Verfassers.

2 »Glühende Strahlen schiessen durch dieses Reiches tiefe Nacht, und wir werden Riesenschatten gewahr, die auf- und abwogen, enger und enger uns einschliessen, und alles in uns vernichten, nur nicht den Schmerz der unendlichen Sehnsucht, in welcher jede Lust, die, schnell und in jauchzenden Tönen emporgestiegen, hinsinkt und untergeht, und nur in diesem Schmerz der Liebe, Hoffnung, Freude in sich verzehrend, aber nicht zerstörend, unsre Brust mit einem vollstimmigen Zusammenklange aller Leidenschaften zersprengen will, leben wir fort und sind entzückte Geisterschar.« [Ernst Theodor Amadeus Hoffmann:] Recension, in: Allgemeine musikalische Zeitung I2 (I8IO), Nr. 40, Sp. 630-642 und Nr. 4I, Sp. 652-659, hier Sp. 632 f.

3 Äußerung Brahms' gegenüber dem Dirigenten Hermann Levi, zit. nach Max Kalbeck: Johannes Brahms, Berlin I904, Bd. I, S. 165 .

4 »Die letzte Spmphonie Beethovens ist die Erlösung der Musik aus ihrem eigensten Elemente heraus zur allgemeinsamen Kunst. Sie ist das menschliche Evangelium der Kunst der Zukunft. Auf sie ist kein Fortschritt möglich, denn auf sie unmittelbar kann nur das vollendete Kunstwerk der Zukunft: das allgemeinsame Drama, folgen, zu dem Beethoven uns den künstlerischen Schlüssel geschmiedet hat.« 
wieder das Modell Beethoven auf. Fast ebenso wichtig wie der Vorbildcharakter für Komponistinnen und Komponisten ist allerdings der Ruf, den Beethoven weit über das regelmäßige Konzertpublikum hinaus in der Gesellschaft genießt. Kaum jemand, der nicht das >Schicksalsmotiv < der Fünften oder den Freudenhymnus der Neunten zumindest als akustische Chiffre kennt. Und selbst die Zahl Neun hat - im Rückblick auf Beethoven, aber auch auf Schubert, Bruckner oder Dvořák - einen beinahe mythischen Nimbus erhalten, indem sie trivialpsychologisch als durch die sgroßen Komponisten überbietende Anzahl der Sinfoniekompositionen verklärt wird. Von Gustav Mahler wird sogar überliefert, er habe die vermeintlich schicksalhafte Zahl mit dem Lied von der Erde vermeiden wollen - worauf er zwar diese eigentliche Neunte ebenso wie die offiziell als >Neunte< gezählte Sinfonie überlebte, dann aber dennoch über der Zehnten starb. 5 Doch auch wenn man von diesem Aberglauben absieht - schließlich gibt es ja auch zahlreiche Komponisten, deren Werk weit über neun Sinfonien hinausgeht -, hat die Neunte in der Nachfolge Beethovens im Anspruch zweifellos eine Sonderstellung inne.

Dieser Erwartungshaltung eines epochemachenden Werks stellte sich auch Dmitri Schostakowitsch, als er sich in der Schlussphase des für die Sowjetunion zwar verlust-, aber doch siegreich zu Ende gehenden >Großen Vaterländischen Krieges` an die Komposition seiner Neunten machte. Was die Öffentlichkeit von ihm begehrte, schildert er selbst in seinen von Solomon Wolkow herausgegebenen (und in ihrer Authentizität bis heute umstrittenen) Memoiren: »Man erwartete von mir einen Fanfarenstoß. Eine Ode wurde verlangt. $\aleph^{6}$ Nachdem er bereits zuvor mehrfach Vokalsinfonien vorgelegt hatte, schien ein weiteres Werk mit großem Orchester, Chor und Solisten die einzig würdige Möglichkeit, den sich abzeichnenden Sieg der sozialistischen Sowjetrepubliken zu feiern.

Schostakowitsch fügte sich zunächst diesen Vorstellungen und erklärte sich Ende I944 bereit, eine solche Apotheose zu komponieren, »wenn ich nur einen geeigneten Text fände und wenn ich nicht Angst hätte, man würde mir unbescheidene Analogien vorwerfen. «7 Von einem Vorwurf der Unbescheidenheit konnte keine Rede sein, im Gegenteil:

»Man erwartete eine majestätische, den Mut und Heroismus des Siegervolks feiernde Sinfonie. Ein solches Werk sollte die Trias der Kriegssinfonien Schostakowitschs würdig abschließen. Die Lenin-

Richard Wagner: Das Kunstwerk der Zukunft, Leipzig 1850, S. 94; vgl. auch ders.: Oper und Drama, hg. und komm. von Klaus Kropfinger, Stuttgart 2000, etwa S. 74, 302 f.

5 Richard Specht: Gustav Mahler, Berlin/Leipzig I913, S. 355.

6 Die Memoiren des Dmitri Schostakowitsch, hg. von Solomon Wolkow, München 2003, S. 228.

7 Zit. nach Michael Koball: Pathos und Groteske. Die deutsche Tradition im spmphonischen Schaffen von Dmitri Schostakowitsch, Berlin I997 (Studia Slavica Musicologica, Bd. Io), S. I92. 
grader Sinfonie war dann eine harte Chronik des Krieges, die Achte ein trauererfüllter Klagegesang und die Neunte der Triumph des Sieges. ${ }^{8}$

Schostakowitsch begann also im Winter I944/45, noch vor Kriegsende, mit der Komposition einer heroischen Sinfonie und spielte erste Entwürfe sogar einigen Freunden vor, die sie als »kraftvoll und siegreich « beschrieben. ${ }^{9}$ Allerdings stockte die Arbeit bald; »den Komponisten quälten Zweifel und Unsicherheit. « ${ }^{\text {IO }}$ Als Schostakowitsch dann im Sommer I945, nun nach Kriegsende, den ersten Versuch definitiv zur Seite legte und stattdessen eine neue, klassizistisch orientierte und über weite Strecken verspielt gehaltene Neunte Sinfonie komponierte, wusste er genau, dass er sich damit den Erwartungen des ganzen Landes entzog und Publikum und Parteiobere im besten Fall erstaunen, viel wahrscheinlicher aber zumindest letztere gegen sich aufbringen würde: »zum Zeitpunkt ihres Erscheinens muss diese subtile musikalische Mischung aus Tragödie, Lyrik, Ironie und Groteske nicht nur als unverantwortlicher Unfug, sondern auch als direkte Herausforderung Stalins erschienen sein. $\ll^{\mathrm{II}}$

Dabei war Josef Stalin nach dem Krieg erst recht unangreifbar geworden, hatte doch die Sowjetunion unter seiner Führung die Angriffe Nazi-Deutschlands abgewehrt und gleichzeitig an Einfluss und Autorität in der Weltpolitik gewonnen: »Er konnte sich mit Recht als einer der Herren der Welt betrachten. Jetzt erwartete er, dass seine neue Rolle und Machtposition in großen Kunstwerken, die seines Genies würdig waren, besungen würde. « ${ }^{\mathrm{I} 2}$ Diese Huldigung war ihm geradezu ein persönliches Anliegen: »Man wusste auch, dass Stalin sich um die Kunstproduktion persönlich sorgte (auch darum, ob sie seinen Ruhm mehrte).«3

Schostakowitsch betont in seiner Charakterisierung zudem die maßlose Überheblichkeit des sowjetischen Führers:

»Stalin hat ganz gewiß nie an seiner Genialität und seiner Größe gezweifelt. Doch als der Krieg gegen Hitler gewonnen war, schnappte er vollends über. Er war wie der Frosch in der Fabel, der sich zur Größe des Stiers aufblies. Mit dem einen Unterschied, daß auch seine gesamte Umgebung den Frosch Stalin für einen Stier hielt und ihm die entsprechenden Ehren erwies. «4

8 Natalja Walerewna Lukjanowa: Dmitri Dmitrijewitsch Schostakowitsch, Berlin I982, S. I43.

9 Koball: Pathos und Groteske, S. I92. Das Fragment eines Allegro non troppo, das wie die spätere Neunte in Es-Dur steht, wurde 2003 wiederentdeckt und 2006 in Moskau uraufgeführt; vgl. Dmitri Shostakovich [Werkverzeichnis], Hamburg ${ }^{2}$ 20II.

10 Lukjanowa: Dmitri Dmitrijewitsch Schostakowitsch, S. I43.

11 Solomon Wolkow: Stalin und Schostakowitsch. Der Diktator und der Künstler, Berlin 2004, S. 3I4.

12 Ebd., S.3І2.

13 Bernd Feuchtner: »Und Kunst geknebelt von der groben Macht«. Dimitri Schostakowitsch. Künstlerische Identität und staatliche Repression, Kassel 2002, S. I52.

14 Memoiren, S. 229. 
Stalins Launen waren gefürchtet und Schostakowitsch hatte sie bereits I936 selbst erfahren, als seine Oper Ladp Macbeth von Mzensk vermutlich auf Stalins Geheiß zu »Chaos statt Musik« erklärt und in der Folge faktisch verboten wurde. ${ }^{15}$ Zudem wusste er aus seinem Freundeskreis, dass ein solches In-Ungnade-Fallen nicht nur den künstlerischen, sondern auch den realen Tod bedeuten konnte. Zahlreiche Weggenossen des Komponisten wurden vom Geheimdienst abgeholt und tauchten nie wieder auf. Ungeachtet dieser Gefahren sah sich Schostakowitsch außerstande, die Erwartungen der Öffentlichkeit, ja Stalins persönlich zu erfüllen: »Ich konnte keine Apotheose auf Stalin schreiben, konnte es einfach nicht. Mir war klar, worauf ich mich einließ, als ich die Neunte schrieb. ${ }^{\mathrm{I} 6}$

Angesichts der Erwartungen an eine monumentale Siegessinfonie war das Publikum der Uraufführung, die am 3. November 1945 mit den Leningrader Philharmonikern unter Jewgeni Mrawinski stattfand, einigermaßen irritiert. »Wie hätten sie ein choralinstrumentales, Beethoven >über-Mahlerndes $<$ Sieges-Monument umjubelt. Statt dessen wurde ihnen dieses ulkige Opus beschert. «77 Weder die verordnete Feierlichkeit noch das Versprechen von Chor und Solisten wurde eingelöst, stattdessen erklang eine über weite Strecken leichte und spielerische Musik, unterbrochen von zwei tragischen Intermezzi. Die Reaktionen waren denn auch durchmischt. Ein Großteil des Publikums war rasch vom Werk eingenommen und »charmed $«,{ }^{18}$ besonders die offiziellen Stimmen waren aber kritisch. So schrieb der Komponist Marian Kowal: »Wie sollte man darauf gefasst sein, dass ausgerechnet von Schostakowitsch keine hochinspirierte Symphonie über den Sieg zu erwarten war? «9 Auch Stalin soll laut Wolkow sehr ungehalten reagiert haben. Er hielt das Werk für »volksfeindlich«, da es sich der offiziell verordneten »nationalen Freude« widersetzte, ${ }^{20}$ und drohte: »Während des Krieges hatten wir nicht die Zeit dafür, aber jetzt werden wir uns auf angemessene Weise um euch alle küm-

Zum gleichnamigen Artikel in der Prawda vom 28. Januar 1936 und den Umständen vgl. etwa Krzysztof Meyer: Schostakowitsch. Sein Leben, sein Werk, seine Zeit, Bergisch Gladbach I995, S. 22I-236. Memoiren, S. 230.

17 Boris Schwarz: Musik und Musikleben in der Sowjetunion von I9IJ bis zur Gegenwart, Wilhelmshaven I982 (Taschenbücher zur Musikwissenschaft, Bd.67), Bd. I, S.347.

18 Roy Blokker/Robert Dearling: The Music of Dmitri Shostakovich. The Symphonies, London I979, S. 32. Auch die Kritiker Schostakowitschs mussten dies anerkennen: »Die Lobhudelei um Schostakowitsch erreichte mit den Aufführungen seiner Neunten Sinfonie ihren Höhepunkt.« Marian Kowal: Der Schaffensweg des Dmitri Schostakowitsch, urspr. in: Sovetskaja muzpka, H. 2, 3 und 4/1948, zit. nach »Volksfeind Dmitri Schostakowitsch«. Eine Dokumentation der öffentlichen Angriffe gegen den Komponisten in der ehemaligen Sowjetunion, hg. von Ernst Kuhn, Berlin I997 (Opyt, Bd.3), S. I88. 
mern. ${ }^{2 \mathrm{II}}$ In der Tat war die mittelfristige Reaktion auf das Werk beziehungsweise auf Schostakowitschs Schaffen allgemein fatal. Mit einem Beschluss des Zentralkomitees wurde der Komponist I948 als >Formalist ‘ gebrandmarkt und stark angegriffen. ${ }^{22}$ In den Memoiren schildert er die Vorgänge folgendermaßen:

»Mit dieser Neunten wurde es ganz schlimm. Ich wusste natürlich, dass der Schlag unvermeidlich war. Aber vielleicht hätte er mich später getroffen, vielleicht wäre er nicht ganz so hart ausgefallen, wenn die Neunte nicht gewesen wäre. [...] Aber so absurd es auch klingen mag, Stalin kümmerte sich um ihm vorenthaltene Huldigungen sehr viel mehr als um die Angelegenheiten des Landes. $\ll^{23}$

Die Interpretationen der Sinfonie gingen allerdings auch in andere Richtungen; so sprach Boris Schwarz vom »vielleicht überschwänglichste[n] Tribut an die Siegesfreude« und einem »freudige[n] Seufzer der Erleichterung «. ${ }^{24}$ »Nur der Sieg konnte dieses fröhliche, elegante, aber auch ironische Werk ins Leben rufen, das durch die Vollkommenheit und Geschliffenheit seiner Sprache, durch seine frappierende, fast kindlich-biedere Offenheit beeindruckt.« ${ }^{25}$ Dass auch ein Großteil des Publikums die Sinfonie bald schätzte, lag laut Wolkow daran, dass Schostakowitschs Musik »die versteckten Gefühle der `Unterschichten aus[drückte]«. ${ }^{26}$ Rasch wurde das Stück zum Erfolg und kam auch in Europa und Amerika zur Aufführung. Dabei stand etwa in der Erstaufführung in den USA neben der Neunten und den Bildern einer Ausstellung von Modest Mussorgski auch Beethovens Sinfonie Nr. 3 auf dem Programm. ${ }^{27}$

Letzteres Werk kann dabei mit Fug und Recht als historisches Gegenstück zu Schostakowitsch Werk gesehen werden, wie Hartmut Schick betont:

»Mit dem Rückgriff auf einen archaisch-klassizistischen Tonfall und auf extrem knappe, quasi Haydnsche Proportionen kehrt Schostakowitsch aber beim Übergang von der 8. zur 9. Symphonie genau die Entwicklung um, die in der Geschichte der Symphonie mit Beethovens Eroica begonnen hat, der ersten großen Symphonie im Sinne des ig. Jahrhunderts. Gattungsgeschichtlich ließe sich insofern Schostakowitschs Neunte bereits wegen ihrer frappanten Kürze als eine Art Anti-Eroica verstehen, quasi als Zurücknahme der Eroica. $\aleph^{28}$

21 Grigori Marjamow: Kremlewski zensor. Stalin smotrit kino, Moskau I992, S. 74, zit. nach Wolkow: Stalin und Schostakowitsch, S.3I5.

22 Siehe dazu etwa »Volksfeind Dmitri Schostakowitsch«.

23 Memoiren, S. $229 f$.

24 Schwarz: Musik und Musikleben in der Sowjetunion, S. 346.

25 Lukjanowa: Dmitri Dmitrijewisch Schostakowtisch, S. I44f.

26 Wolkow: Stalin und Schostakowitsch, S.3I4.

27 Manashir Iakubov: Dmitri Shostakovich. The Ninth Symphony. Score [Nachwort], in: Dmitri Shostakovitch. Symphony No. 9, hg. von Manashir Iakubov, Moskau 2005 (New Collected Works, Bd. 9), S. I22.

28 Hartmut Schick: Die unpolitisch Heitere? Versuch einer Neuinterpretation von Schostakowitsch IX. Symphonie von I945, in: Schostakowitsch und die Spmphonie. Referate des Bonner Spmposions 2004, hg. von 
Zum Aufbau Dmitri Schostakowitsch setzte sich in seinen Kompositionen jeweils sehr bewusst mit der Tradition und seinen Vorgängern auseinander. So berichtet er in den Memoiren über eine >Lektion`, die er vom russischen Schauspieler und Regisseur Wsewolod Meyerhold gelernt habe: »Aufjede Arbeit musst du dich vorbereiten. Eine Menge Musik durchgehen, vielleicht bei den Klassikern nach etwas Ähnlichem suchen und dann versuchen, es besser zu machen oder wenigstens auf deine eigene Art. ${ }^{29}$ Bezogen auf seine Vorgänger und dennoch in eigener Art und Weise hat Schostakowitsch auch seine Neunte gestaltet. Wo die Öffentlichkeit eine monumentale Sinfonie zum Ende des Zweiten Weltkriegs erwartete, entschied er sich im Gegenteil für eine »Anti-Neunte«: eine sehr kurze und zumindest auf den ersten Blick >leichtgewichtige «, klassizistische Sinfonie. Allerdings sind neben offensichtlichen allgemeinen Anklängen an die klassische Epoche eben nicht nur Bezüge zu Beethovens Neunter, sondern insbesondere auch zu dessen Eroica auszumachen, angefangen bei der vorgezeichneten Tonart Es-Dur, die in der Klassik als heroische und Militärtonart gehandelt wurde: »Die Tonart es dur wird auch zuweilen der Feldton genannt wird, weil die bey der eigentlichen Kriegsmusik gewöhnlichen Instrumente, als Trompeten, Clarinetten, Hörner u. s. w. nach dieser Tonart mensurirt sind. 30 $^{\circ}$

Gleichzeitig grenzt er sich damit von der für festliche Musik gebräuchlicheren `Jubeltonart $<$ D-Dur ab, wie sie Beethoven im Finale der Neunten verwendet und wie sie seit dem Barockzeitalter etwa für die Vertonung des »Te Deum« oder anderer glanzvoller Texte üblich war. Schostakowitsch selbst hatte sie - ebenso wie das Schema sper aspera ad astra - in seiner Fünften Sinfonie eingesetzt und dort mit nicht enden wollendem Dur-Jubel auf die Spitze getrieben und persifliert. ${ }^{3}$

Im großformalen Aufbau fallen vor allem die fünf Sätze der Sinfonie auf. Im Gegensatz zur klassischen Viersätzigkeit schiebt Schostakowitsch vor dem Finale einen weiteren Satz ein, erhält damit die Satzfolge schnell (Allegro) - langsam (Moderato) - schnell (Presto) - langsam (Largo) - schnell (Allegretto/Allegro) und somit starke Kontraste zwischen den einzelnen Sätzen. Die drei letzten Sätze gehen allerdings ohne Unterbruch

Hartmut Hein und Wolfram Steinbeck, Frankfurt a. M. u. a. 2007 (Bonner Schriften zur Musikwissenschaft, Bd. 7), S. 2II-224, hier S. 2I4f.

29 Memoiren, S. I59.

30 Heinrich Christoph Koch: Kurzgefaßtes Handwörterbuch der Musik für praktische Tonkünstler und für Dilettanten, Leipzig I807, S. I42.

Vgl. etwa Hans-Joachim Hinrichsen: »Das ist doch keine Apotheose.« Warum ist Schostakowitschs V. Symphonie so mißverständlich ?, in: Schostakowitsch und die Spmphonie. Referate des Bonner Symposiums 2004, hg. von Hartmut Hein und Wolfram Steinbeck, Frankfurt a. M. 2007 (Bonner Schriften zur Musikwissenschaft, Bd. 7), S. I37-I59. Vgl. zudem das Kapitel zur Fünften bei Uta Schmidt: Kompositionen mit doppeltem Boden. Musikalische Ironie bei Erik Satie und Dmitri Schostakowitsch, Schliengen 20I4 (Kontext Musik, Bd.3), S. 270-283. 
ineinander über, weshalb der vierte Satz auch als langsame Einleitung zum Finale gesehen werden kann. ${ }^{32}$

Ebenfalls nicht nach klassischem Schema, allerdings auch nicht völlig ungewohnt, sind die aufeinander bezogenen Grundtonarten der Sätze - wobei allerdings je nach Erklärungsversuch ein anderer Satz ausschert. So finden sich, wenn man den zweiten Satz ausblendet, mit Es, G, b und Es 33 die Töne des Grunddreiklangs als Ausgangspunkt für die tonalen Verhältnisse zwischen den Sätzen. Der zweite Satz - traditionsgemäß stünde er in c-Moll, also der Mollparallele der Grundtonart - fällt dabei mit h-Moll völlig aus dem Rahmen. Dieses h-Moll ließe sich aber mit einer anderen Deutungsmöglichkeit erklären, wenn man den vierten Satz eben als Einleitung für den fünften auffasst und ihn für die Tonarten-Verhältnisse beiseitelässt. Die Sätze stünden dann in einem Großterzabstand zueinander: Es (Dis), h, G und wieder Es. Dieser zweite Erklärungsversuch ist allerdings sehr hypothetisch, eine Deutung der Tonart h-Moll als Ersatz für das zu erwartende c-Moll liegt wohl näher.

Demgegenüber ist der Tonartenplan bei Beethoven selbstredend viel einfacher gehalten - wobei allerdings auch dieser sich stellenweise einen Spaß daraus macht, allzu klare tonale Verhältnisse zu vermeiden oder aber zu verwischen. So begnügt sich etwa die Dritte Sinfonie mit zwei Grundtonarten, nämlich mit Es-Dur (im ersten, dritten und vierten Satz) und dem oben vermissten c-Moll (im zweiten, langsamen Satz). Überraschend ist dort allerdings der Beginn des vierten Satzes, der mit einem Unisono der Streicher in g-Moll beginnt, das sich dann zur Dominante B-Dur weiterentwickelt, bevor endlich die Tonika Es-Dur aufscheint. Noch um einiges deutlicher findet sich dieses Versteckspiel zu Beginn seiner Neunten Sinfonie: Sechzehn Takte lang erklingt die leere Quinte $a$ - e, gewinnt durch Einwürfe an rhythmischen Konturen und mündet schließlich in einem Fortissimo-Ausbruch der Haupttonart d-Moll. Erst rückblickend wird also die Funktion des Beginns als Dominante klar. Beim zweiten Anlauf, beginnend mit der Quinte $d$ - $a$, wendet sich das Ganze nicht etwa nach g/G (entsprechend der ersten Stelle) oder zurück zur Dominante A-Dur, sondern überraschend nach B-Dur. Im Blick auf das ganze Werk werden dadurch die Haupttonarten exponiert: Während der zweite Satz in d-Moll und D-Dur steht, findet sich im dritten wieder B-Dur - und auch der letzte Satz basiert hauptsächlich auf diesen drei Tonarten.

Doch zurück zu Schostakowitschs Neunter, die bereits mit dem quasi nach Lehrbuch gestalteten Sonatensatz - inklusive Wiederholung der Exposition ${ }^{34}$ - ihre Orientierung

32 Vgl. etwa Karen Kopp: Form und Gehalt der Spmphonien des Dmitrij Schostakowitsch, Bonn I990, S. 291.

33 Großschreibung entspricht hier und in Folge den Dur-, Kleinschreibung den Moll-Tonarten. Ebenfalls in (kursivierten) Kleinbuchstaben angegeben sind Einzeltöne.

34 Die Wiederholung der Exposition ist im I9. Jahrhundert nur mehr selten ein Thema. Beethoven selbst 
an klassischen Vorbildern (und hier somit eigentlich weniger an Beethoven) verrät und diese fast sklavisch befolgt. Dadurch entsteht ein starker Kontrast zwischen einer überschäumenden und spielerischen Musik sowie der unnachgiebigen Form - wobei letztere die Entwicklungen der Musik fast gewalttätig wieder zum >richtigen` Ergebnis zurückzwingt. So steht beispielsweise das Seitenthema wie gewohnt in B-Dur, nachdem sich die Musik zuvor deutlich gegen A-Dur hinbewegt hatte. ${ }^{35}$ Die Form dominiert so die Komposition; als übergeordnete Instanz zwingt sie ihr ein Korsett auf. Dies wohl nicht grundlos: »Auch dort, wo die Form quasi als Zitat steht (9/I), hat sie eine Bedeutung, die über den Form-Sinn hinausgeht. ${ }^{36}$ Einerseits mag diese starre Form, die eine freie Entwicklung der Musik behindert, ein Abbild für die Situation Schostakowitschs in Russland sein - in gewissen Grenzen genoss er eine Art Narrenfreiheit, wurde jedoch immer wieder in die Schranken gewiesen - andererseits wird ihr Einsatz als Zurücknahme von Beethoven gedeutet, der als Erneuerer gesehen wird, der das Korsett der Form zu lockern begann. ${ }^{37}$

Dreiklangsmelodik und >falscheく Töne Der Beginn des Hauptthemas orientiert sich in seiner Dreiklangsbrechung ebenfalls an der Klassik - Stichwort `Mannheimer Rakete <-, kehrt allerdings deren Bewegungsrichtung um. Es ginge angesichts des Charakters der Musik wohl zu weit, die Form dieser Figur auf das ähnlich gelagerte, aber ungleich dramatischere Hauptthema des ersten Satzes von Beethovens Neunter zurückzuführen, wichtiger ist, dass die vermeintlich heroische Rakete und mit ihr die Hörerwartung einer sinfonischen Apotheose mit den ersten Tönen gleichsam abstürzt. Viel bezeichnender ist die Fortführung des Themas hin zu einem mit einem mit Akzent und Triller versehenen (und zudem durch Celli und Bässe verstärkten) ges im vierten Takt. Unweigerlich

hatte sie etwa in seiner Appassionata weggelassen und dies auch für den Kopfsatz der Eroica geplant. Aufgrund der überlangen Durchführung und Coda entschied er sich in letzterem Fall schließlich doch für eine Wiederholung; vgl. Peter Schleuning: 3. Symphonie Es-Dur Eroica op.55, in: Beethoven. Interpretationen seiner Werke, hg. von Albrecht Riethmüller, Carl Dahlhaus und Alexander Ringer, Laaber i994, Bd. I, S.386-400, hier S.395f.

Gojowy deutet das e nicht als Dominante zu a, sondern als Leitton zu f: »Schostakowitsch erreicht diese Dominanttonart sozusagen nicht über die normale Treppe, sondern gewissermaßen an der Dachrinne entlang durchs Fenster.« Detlev Gojowy: Schostakowitsch und der »Theateroktober«. Theatralische Strukturen der Harmonik Schostakowitschs, in: Bericht über das Internationale DmitriSchostakowitsch-Spmposion Köln I985, hg. von Klaus Wolfgang Niemöller, Regensburg I986 (Kölner Beiträge zur Musikforschung, Bd. I50), S. 540-552, hier S.549. Allerdings wirkt das e durch den bestätigenden Oktavsprung kaum als Leitton.

36 Kopp: Form und Gehalt, S. IO2.

37 Schick: Die unpolitisch Heitere?, S. 2I5. 
denkt man hier an die Note der Musikgeschichte, wie sie Richard Wagner einmal gegenüber Cosima bezeichnet hat:

»Am Morgen hatte mir R. zugerufen: ‘weißt Du wie die Note der ganzen neueren Musik heisst? Sie heisst Cis, es ist das Cis des ersten Thema der Eroica, [w]er hatte vor Beethoven, wer hat nach ihm diesen Seufzer in der völligen Ruhe eines Thema's ausgestossen? « «8

Inmitten des wiegenden, in Dreiklangsbrechung aufsteigenden Hauptthemas von Beethovens Dritter sinkt die Linie der Celli dort im siebten Takt überraschend über $d$ zu cis. Während bei Beethoven das fremde cis nur noch verhältnismäßig kurz als Durchgangstonart in der Reprise aufscheint (Des-Dur, Takt 4I6-4I9 beziehungsweise 557-56I), baut Schostakowitsch sein ges (das im Übrigen einfach als Vorhalt zu f als Quintton der Dominante B-Dur aufgelöst wird) prominent in den Formplan des Satzes ein und beginnt die Durchführung (Ziffer II) in Ges-Dur. ${ }^{39}$
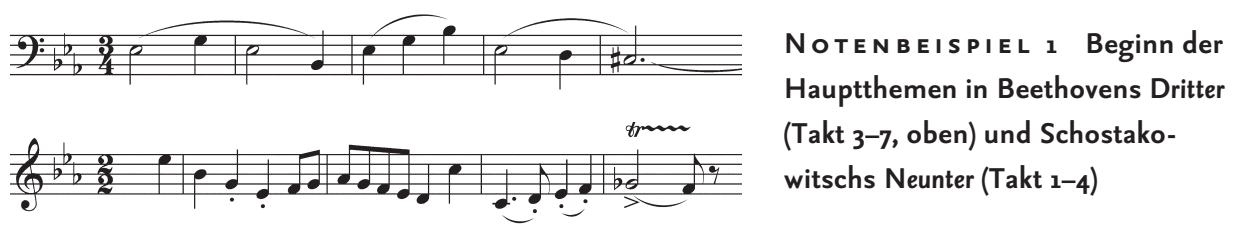

In dieser Durchführung fällt ein Aspekt ins Auge, der vermutlich eher aus kräftemäßigen Überlegungen gewählt wurde, dennoch stutzt man, wenn man sich an Beethovens Dritte Sinfonie erinnert: Außergewöhnlich für seine Zeit schrieb dieser dafür nämlich drei (Natur-)Hörner in derselben Tonart vor. Üblich waren sonst meist Hornpaare, also zwei oder allenfalls vier Hörner, in letzterem Fall dann aber oft in zwei verschiedenen Tonarten, um bei Modulationen mehr Einsatzmöglichkeiten für die auf den Vorrat der Naturtöne beschränkten und allenfalls durch klanglich veränderte Stopftöne erweiterbaren Instrumente zu haben. Mit dem modernen Ventilhorn ist Schostakowitsch selbstredend keinen solchen Einschränkungen mehr unterworfen; interessant ist allerdings, dass sich die Hörner für die ersten I4 Takte der Durchführung zunächst nur mit drei Stimmen abwechseln. Erst für die Akkorde ab Takt ro2 erklingen schließlich alle vier Hörner gemeinsam.

Die Hörner oder besser das zweite Horn spielt auch in einer nächsten Episode um ,falscheく Töne eine Rolle, die dem Beethoven-Freund Ries laut eigener Aussage beinahe Schläge des Komponisten eintrug:

38 Zit. nach Cosima Wagner: Die Tagebücher, Bd.I, ediert und komm. von Martin Gregor-Dellin und Dietrich Mack, München I976, S. III5.

39 Der Vollständigkeit halber sei darauf hingewiesen, dass auch im ersten Satz der Eroica (Takt 330 ff.) eine kurze Episode in Ges-Dur nicht fehlt. Ein Zusammenhang der beiden Stellen fällt aber ansonsten nicht ins Auge. 
»In dem nämlichen Allegro [I. Satz der Eroica] ist eine böse Laune Beethoven's für das Horn; einige Tacte, ehe im zweiten Theile das Thema vollständig wieder eintrit [sic], läßt Beethoven dasselbe mit dem Horn andeuten, wo die beiden Violinen noch immer auf einem Secunden-Accorde liegen. Es muß dieses dem Nichtkenner der Partitur immer den Eindruck machen, als ob der Hornist schlecht gezählt habe und verkehrt eingefallen sei. Bei der ersten Probe dieser Symphonie, die entsetzlich war, wo der Hornist aber recht eintrat, stand ich neben Beethoven, und im Glauben, es sei unrichtig, sagte ich: >der verdammte Hornist! kann der nicht zählen? - Es klingt ja infam falsch! Ich glaube, ich war sehr nah daran, eine Ohrfeige zu erhalten. - Beethoven hat es mir lange nicht verziehen. « ${ }^{\circ}$

Mit falschen Einsätzen spielt auch Schostakowitsch, wobei der Part des >Sünders` hier der Soloposaune zukommt, einem Instrument, das er in seinen Memoiren - wenn auch wohl eher ironisch - als »verdächtig« apostrophiert. ${ }^{4 \mathrm{I}}$ Bereits in der Einleitung setzt die Posaune nach einer »Dominante«E (zu A-Dur) mit einem Quartauftakt $f$ - $b$ das zwar formal-traditionell richtige, aber einigermaßen unerwartete B-Dur für das Seitenthema durch, das als marschartiges Piccolo-Thema mit Trommelbegleitung daherkommt. In der Reprise dann (also im Unterschied zu Beethoven nicht vorher) folgt der Auftakt der Posaune bereits inmitten des Hauptthemenkomplexes (ein Takt vor Ziffer I9) und wird aufgrund der ausbleibenden Reaktion der übrigen Instrumente dann auch ostentativ noch fünfmal in kürzeren oder längeren Abständen wiederholt. Im Gegensatz zum vorsichtigen Einsatz des Horns bei Beethoven (im Pianissimo), kennt die Posaune dabei keine falsche Scheu und versucht ihr Glück jeweils im Forte oder Fortissimo. Erst beim nun regulären sechsten Einsatz (Ziffer 2I) schließt sich das übrige Orchester - hier nun die Solovioline, begleitet vom tiefen Blech und der kleinen Trommel - dem kadenzierenden Impuls der Posaune an. Zusätzlich zum sowieso schon auf dem »letzten Loch« geblasenen ${ }^{42}$ und karikaturhaft gestalteten Seitenthema ${ }^{43}$ gerät diese Stelle in der Reprise zu veritablem Slapstick. Und sogar in der Coda versucht es die Posaune - nun allerdings im verschämten Piano - noch einmal mit einem Quartauftakt (Takt 2I7); womöglich wollte sie die Reprise analog zur Exposition versehentlich wiederholen ...

$>$ Valse triste< und Zirkusmusik Einen starken Kontrast zu den ungestümen Klängen des ersten bildet der zweite Satz. Mit dem Wechsel in eine Molltonart und der völligen Zurücknahme der Instrumentation auf Soloklarinette und begleitende Pizzicati der

Franz Gerhard Wegeler/Ferdinand Ries: Biographische Notizen über Ludwig van Beethoven, Koblenz I8 38 , S. 79 .

41 Memoiren, S. I95.

42 Vgl. auch Schick: Die unpolitisch Heitere?, S. 2I8.

43 Das Thema soll sich auch auf ein Motiv beziehen, das als Karikatur des »Yankee«, also des Amerikaners diente; siehe dazu Marina Sabinia: Das sinfonische Schaffen von Schostakowitsch und dessen Beziehungen zu Film und Theater, in: Bericht über das Internationale Dmitri-Schostakowitsch-Spmposion Köln 1985 , S. 375-384, hier S. 380 f. 
tiefen Streicher schafft Schostakowitsch eine sehr intime Atmosphäre. Wiederum steht am Anfang eine Dreiklangsbrechung, allerdings in der unerwarteten Tonart h-Moll. Es scheint, als wäre das von Beethoven verwendete c-Moll um einen Halbton abgerutscht, vom Heldengedenken mit Trauermarsch der Eroica zu einem Klagelied in der Folge von Bachs h-Moll-Messe oder von Tschaikowskis Sechster Sinfonie geworden. ${ }^{44}$ Der solistische Einsatz der Klarinette und der gedehnte Quintton verleihen dem Satzbeginn einen schwerelosen, in Verbindung mit den kleinen Sekundschritten zugleich klagenden Charakter. Dazu passt auch das jeweils sanfte Anstoßen der Melodie durch die Pizzicati der Streicher. Sehr frei und beinahe improvisiert wirkt die zaghafte Fortspinnung der >unendlichen $<$ Melodie des Themas. Der Dreiertakt erinnert an eine $>$ Valse triste $<$ die allerdings durch eingeschobene Viervierteltakte immer wieder ins Stocken gerät. Dabei taucht in Verbindung mit diesen gedehnten Takten und dem Ende der Phrase jeweils das Intervall der fallenden Quarte auf, das allerdings im Gegensatz zum Quartsprung des ersten Satzes hier eher von resignierendem Charakter ist. Der Schluss des Satzes wiederum bietet eine erneute Parallele zur Eroica: Während das Piccolo den Themenkopf übernimmt, werden die begleitenden Streicherpizzicati immer leiser und seltener, der Satz >erstirbt‘. Derweil fehlt bei Beethoven zwar im Gegensatz zu Schostakowitsch die konkrete Spielanweisung »morendo«; indem er die melodieführenden ersten Geigen allerdings »sempre più piano« spielen lässt und das Thema mit immer mehr Pausen durchsetzt, erreicht er quasi denselben Effekt. Während dort mit dem Topos des Marschs aber eine Art ১offizielles` Heldenbegräbnis stattfindet, handelt es sich bei Schostakowitsch um eine sehr persönliche und zurückhaltende Form von Trauer, die sich angesichts des eben überstandenen Zweiten Weltkriegs und insgesamt im Kontext der stalinistischen Herrschaft wohl nicht als Denkmal für einen großen Helden, sondern als Erinnerung an die zahllosen Opfer lesen lässt.

War bereits der zweite Satz eher lose mit Beethoven in Verbindung zu bringen, trifft das für den dritten erst recht zu. Als »sudden flash of lightning «45 bildet er erneut einen starken Kontrast zu den vorhergehenden Sätzen. Das erste Thema in den Holzbläsern, von Koball als »munteres Jahrmarktgedudel « bezeichnet, ${ }^{46}$ ist dabei geprägt durch die abwechselnden staccato-Dreiklangsbrechungen und legato-Sechzehntelläufe. Durch das rasche Tempo, die regelmäßige achttaktige Periodisierung - die mit der Zeit mehr und mehr durcheinandergerät - und den Sechsachteltakt erhält der Satz einen ausgeprägten Scherzocharakter. Meyer schildert, dass Schostakowitsch bei den Proben zur Uraufführung »nervös im leeren Saal der Philharmonie auf und ab ging und dabei ständig >Zirkus,

44 Schick: Die unpolitisch Heitere?, S. 220.

45 Ivan Martynov: Dmitri Shostakovitch. The Man and his Work, New York 1947, S. I5I.

46 Koball: Pathos und Groteske, S. I96. 
Zirkus $<$ vor sich hersagte, als wollte er damit dem Dirigenten den Charakter seiner Musik begreiflich machen. «47 Insbesondere das von einer Blechkaskade eingeleitete Trio in fis-Moll wurde dabei als »Zirkusmarsch « bezeichnet. ${ }^{8}$ Grund dafür ist wohl insbesondere die fanfarenartige Trompetenmelodie, die - später mit tiefen Streichern, Hörnern und Fagotten instrumentiert - ins Bedrohliche abgleitet. Gerade diese Trompetenmelodie kann allerdings durchaus als Parallele zu Beethovens Eroica gesehen werden, übernehmen doch auch in deren Scherzo-Trio die Blechbläser - konkret die Hörner - führende Funktion. Und während es durchaus üblich war, Trios in Holzbläserbesetzung (teilweise ergänzt durch die Hörner) zu instrumentieren, stellen solche melodischen Einsätze keineswegs den Standard der Blechbläserbehandlung zu Beethovens Zeit dar.

Selbstverständlich kann auch der Charakter als Scherzo an sich als Reverenz an den Klassiker gesehen werden, gilt er doch als Meister dieser Gattung. Darüber hinaus setzen beide Komponisten auf den Kontrast von staccato-Vierteln/Achteln und legato-Achteln/ -Sechzehnteln im Thema und geben trotz den unterschiedlichen Bezeichnungen als Presto (bei Schostakowitsch) respektive Allegro vivace (bei Beethoven) ähnliche Tempovorgaben: die punktierte Viertel/Halbe zu I26 beziehungsweise II6.

»Oh Freunde, nicht diese Töne« Deutlich näher liegt der Bezug zu Beethoven selbstredend im vierten Satz, der sich durch den nahtlosen Übergang in den fünften Satz wie schon erwähnt auch als langsame Einleitung zum Finale verstehen lässt. ${ }^{49}$ In der Eroica ebenso wie in Beethovens Neunter ist dem letzten Satz eine Einleitung vorangestellt. Wo bei Beethovens Dritter und Neunter Sinfonie nahezu kakophonische Ausbrüche stehen, findet sich bei Schostakowitsch eine Unisono-Passage in Posaunen und Tuba. Die Mittel könnten verschiedener nicht sein, der Effekt aber ist sehr ähnlich. Beide Komponisten schockierten das Publikum: Beethoven mit ungewohnten Klangballungen, Schostakowitsch mit einer grauenerregenden, kahlen Tonleiter. Wo bei den anderen Sätzen bisher meist Parallelen zur Dritten Sinfonie gezogen wurden, bietet sich in diesem aus dem normalen Sinfonieablauf heraustretenden Largo die Neunte als Vergleich an. Auffälligster Bezug ist selbstverständlich das zweimal wiederkehrende Fagottrezitativ, das an das Instrumentalrezitativ der tiefen Streicher beziehungsweise den Einsatz des Solo-Baritons im vierten Satz von Beethovens Werk erinnert. Doch nicht nur zum letzten, auch zum ersten Satz der Neunten finden sich Entsprechungen. So können die doppelt punktierten Viertel der Blechbläser bei Schostakowitsch als Anspielung an das ebenso rhythmisierte Hauptthema bei Beethoven gesehen werden. Wo dieser allerdings wiederum Dreiklangs-

47 Meyer: Schostakowitsch, S.3I7.

48 Kopp: Form und Gehalt, S. 289.

49 Ebd., S.29I. 
brechungen - notabene unisono - spielen lässt, bewegt sich die melodische Linie von Posaunen und Tuba (ebenfalls unisono) zunächst Ton für Ton nach oben, um nach einem überraschenden Einfall des Tritonus mit einem Quintfall und somit in einer Art Kadenz zu schließen. Das so erreichte Unisono- $a$ wird mit dem Einsatz der Trompeten und Streicher unerwartet zur Terz des - in b-Moll regulären - Dominantklangs F-Dur. Eine ähnlich unvermutete Wendung findet sich ebenfalls bei Beethoven.

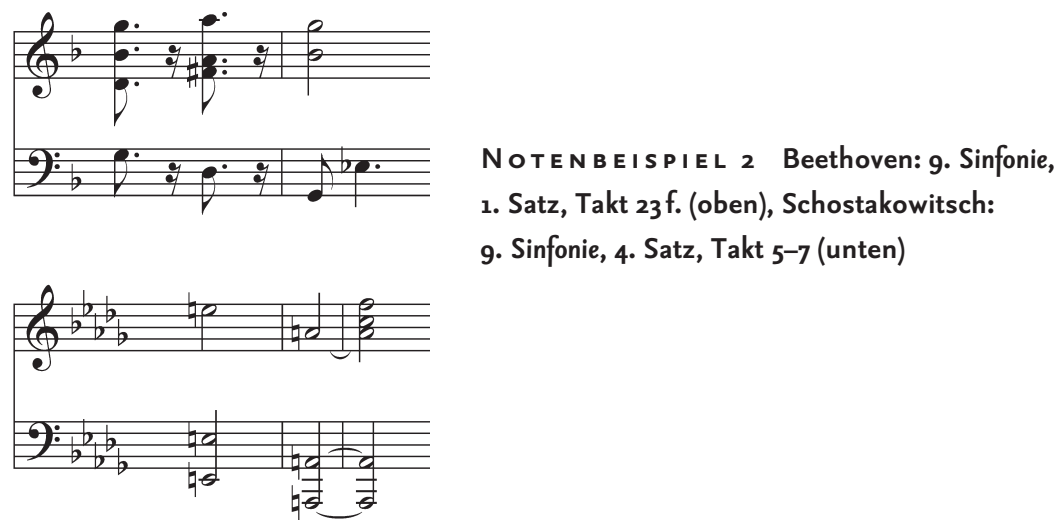

So folgt auf das Thema im ersten Satz der Neunten Sinfonie zunächst eine Kadenz in d-Moll (Takt 2I) und anschließend in g-Moll (Takt 23). Die Celli und Kontrabässe verlassen das g aber sofort wieder zugunsten von es. Da dieser Wechsel im Bass stattfindet, erklingt bei Beethoven ein regulärer Es-Dur-Akkord, während bei Schostakowitsch ein Sextakkord F-Dur über dem Basston a stehenbleibt. Erstaunlich ist allerdings, dass Schostakowitsch über diesen Umweg die >gewöhnliche< Dominante (als Sextakkord) erreicht, während bei Beethoven mit der vertieften zweiten Stufe ein Neapolitaner in Grundstellung erklingt. Den F-Dur-Sextakkord nimmt Schostakowitsch als Ausgangspunkt für ein ausdrucksvolles Fagott-Rezitativ, das entgegen dem Bariton-Solisten in Beethovens Neunter gerade nicht mit »Oh Freunde, nicht diese Töne« gegen Chaos und Rückschau auf Vergangenes ansingt, sondern im Gegenteil von Reflexion und Klage geprägt ist. Wenig erstaunlich, dass es deshalb nicht als flammender Appell, sondern als »Trauerrede am Grab der Gefallenen « 50 oder schmerzliche Antwort auf die »einschüchternde [...] Anklage « $5^{\mathrm{I}}$ des Blechs gehört wurde. Umso erstaunlicher und sunpassender wirkt daher der aus dieser Trauerstimmung hervorgehende letzte Satz, in dem das Fagott (ähnlich wie bei Beethoven der Bariton) unvermittelt selbst »neue Töne« anstimmt und somit implizit eine fröhliche Maske aufsetzt: »The composer [...] puts on a mask and does not reveal his true thoughts on the surface; in any case, the final word remains 
allegorical. Shostakovich tries to avoid the final point. « ${ }^{22}$ Von unterdrücktem Übermut und Galgenhumor ${ }^{53}$ ist denn im Zusammenhang mit diesem Satz auch die Rede, letztlich von nichtmenschlichen Zügen, blitze doch »hier und da in der allgemeinen Fröhlichkeit das unheildrohende Motiv einer sich krampfhaft krümmenden Marionette auf.« 54

Auch in diesem Satz findet sich noch eine Parallele zu Beethoven. So weist bereits Osthoff55 darauf hin, dass sich das Seitenthema bei Schostakowitsch und eine Episode in Beethovens Variationensatz sowohl in Bezug auf die Tonartenverhältnisse als auch in Bezug auf die Themenform ähneln. Während in der Eroica das Thema in der Obermediante erklingt (g-Moll, Takt 2II), steht es bei Schostakowitsch in der Tonikaparallele, also der Untermediante (c-Moll, Takt II7). Das Thema selbst weist mit dem zunächst bekräftigenden Beginn auf der Quinte, den anschließenden absteigenden punktierten Rhythmen und dem Sechzehntelaufstieg zum Nachsatz ebenfalls eine bemerkenswerte Ähnlichkeit auf.

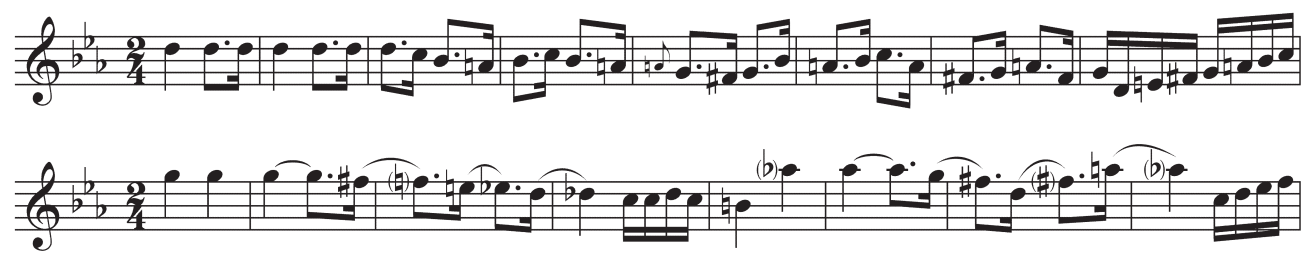

Not e N B E I P I E L 3 Beethoven: 3. Sinfonie, 4. Satz, Takt 211-218 (oben),

Schostakowitsch: 9. Sinfonie, 5. Satz, Takt 117-124 (unten)

Stalin als zweiter Napoleon? Wenn nun jede dieser Parallelen und Bezüge zwischen Beethovens Sinfonien und Schostakowitsch Neunter für sich allein genommen zufällig anmuten mag, sind sie in ihrer Häufung doch erstaunlich und kaum bloß beliebig. Besonders interessant ist auch, dass sich ausgerechnet der >unpassendeく, von der gewöhnlichen Satzfolge abweichende vierte Satz auch in Bezug auf das zitierte Werk von den anderen Sätzen unterscheidet. Als einziger weist er mit Rhythmus, Charakter und Form (Fortissimo-Ausbruch und Instrumentalrezitativ) zurück auf Beethovens Neunte Sinfonie.

Es bleibt die Frage nach der möglichen Bedeutung all dieser Anspielungen. Besonders dieser vierte Satz kann als doppelte Absage an die Ansprüche an eine Neunte ver-

Alexander Ivashkin: Shostakovitch and Schnittke. The Erosion of Symphonic Syntax, in: Shostakovich Studies, hg. von David Fanning, Cambridge 1995, S. 254-270, hier S. 259.

Feuchtner: »Und Kunst geknebelt ...«, S. 153 .

54 Lukjanowa: Dmitri Dmitrijewitsch Schostakowitsch, S. 145.

55 Wolfgang Osthoff: Symphonien beim Ende des Zweiten Weltkriegs. Strawinsky - Frommel - Schostakowitsch, in: Acta Musicologica 60 (1988), S. 62-I04, hier S. 9o. 
standen werden. Anstelle einer »Ode an die Freude«steht hier eine Trauerklage über all jene, die im Krieg und durch das sowjetische Regime getötet wurden. Anstelle eines Aufrufs zum Siegesjubel wird an die Menschen erinnert, die ihr Leben lassen mussten. Die übrigen vier Sätze verweigern sich den Erwartungen an eine Neunte ebenfalls, allerdings auf andere Weise. In ihrem klassizistischen Gewand führen sie die pompösen Feierlichkeiten zum Kriegsende ad absurdum. Schick erinnert daran, dass »die ganze Symphonie also sowohl gegen ihre Tonart, Es-Dur, als auch gegen ihre Nummer, die Neun « geschrieben sei. ${ }^{6}$ Schostakowitschs Werk klingt nicht heldenhaft und auch das Finale ist keineswegs eine Überhöhung im Sinne von >per aspera ad astra (wie bei Beethovens Fünfter), sondern ein vorüberrauschender Kehraus. Mit der Verneinung der von Beethoven gerade in der Dritten Sinfonie errungenen Neuerungen wird Schostakowitschs Neunte in Schicks Worten zu einer »Anti-Eroica« und einem Gegenentwurf zur »ersten großen Symphonie im Sinne des I9. Jahrhunderts «.57 Solche Gegenentwürfe, die die immer massiver aufgeblähte Sinfonik des frühen 20. Jahrhunderts in Frage stellten, hatte es - insbesondere auch auf russischer Seite mit Sergej Prokofjews Symphonie classique (I9I7) oder Igor Strawinskys neoklassizistischen Werken - durchaus bereits zuvor gegeben. Dennoch präsentiert sich die Situation hier unterschiedlich. Diese Zurücknahme steht nach dem Zweiten Weltkrieg und in einem Land mit erheblicher Erwartungshaltung von offizieller Seite hinsichtlich dieser instrumentalen Gattung in einem ganz anderen Licht. Über die bereits praktizierte Reduktion der Mittel hinaus handelt es sich hierbei um eine klare Absage an die Vorstellungen der Machthaber. Die Karikatur und Ironie, die in diesem Werk zum Ausdruck kommen, gelten denn auch nicht etwa der Zeit der Klassik oder gar dem Vorbild Beethoven, sondern sind auf Schostakowitschs Gegenwart und die Erwartungen seiner Zeitgenossen gemünzt. Dass dies ausgerechnet mit jenem Werk gelang, dessen ursprünglicher Widmungsträger Napoleon Bonaparte auf dem Titelblatt durch Beethoven vehement wieder ausgekratzt wurde, nachdem er sich selbst zum Kaiser hatte krönen lassen, ${ }^{8}$ lässt zu guter Letzt noch eine weitere Assoziation zu: Auf Stalin, diesen selbsternannten Heroen, und seine Siegestaten kann und will Schostakowitsch weder eine Eroica noch eine klassische Neunte schreiben. Erst in der Zehnten Sinfonie wird er den Diktator schließlich darstellen - doch auch hier, nach dessen Tod, nicht als Helden, sondern als brutale Fratze. 59

56 Schick: Die unpolitisch Heitere?, S. 224.

57 Ebd., S. 215.

$5^{8}$ In Ries' Erinnerung ist die Reaktion auf die Kaiserkrönung Napoleons gar noch massiver: » Nun wird er auch alle Menschenrechte mit Füßen treten, nur seinem Ehrgeize fröhnen; er wird sich nun höher, wie alle Andern stellen, ein Tyrann werden! Beethoven ging an den Tisch, faßte das Titelblatt oben an, riß es ganz durch und warf es auf die Erde.« Wegeler/Ries: Biographische Notizen, S. 78. 


\section{Inhalt}

Vorwort 8

INTERPRETATION - BEGRIFF, METHODE, PRAXIS

Laure Spaltenstein Interpretation als treue Übersetzung. Zur Frühgeschichte eines vieldeutigen Begriffs I5

Kai Köpp Von der Quelle zur Methode. Zum Entwurf einer historischen Interpretationsforschung $\quad 28$

Manuel Bärtsch >Interpretation‘. Beethovens Sonate A-Dur op. IOI in der Sicht von Eugen d'Albert und Frederic Lamond

Sebastian Bausch Klavierrollen als Interpretationsdokumente. Ein Erfahrungsbericht als Leitfaden für Einsteiger $\quad 7 \mathrm{I}$

Camilla Köhnken Beethoven-Auslegung zwischen Liszts »Deklamationsstil« und Bülows »Vivisektionsversuchen«. Auf den Spuren Liszt'scher Interpretationsideale in Hans von Bülows instruktiver Edition der Klaviersonaten Beethovens $\quad 92$

Neal Peres Da Costa Carl Reinecke's Performance of his Arrangement of the Second Movement from Mozart's Piano Concerto K. 488. Some Thoughts on Style and the Hidden Messages in Musical Notation

II4

Carolina Estrada Bascuñana Enrique Granados's Performance Style.

Visualising the Audible Evidence I5O

Lukas Näf Tempogestaltung in Weberns Sinfonie op. 2I I80

INTERPRETATION - AUFFÜHRUNGSGESCHICHTE

Christoph Moor "Ein so erklärtes Lieblingsstück der hiesigen Kunstfreunde«. Die Rezeptionsgeschichte der Jupiter-Sinfonie in Beethovens Wien

Luisa Klaus Objektive Bruckner-Interpretation? Zur Aufführung der Trio-Entwürfe für die Neunte Sinfonie I940 205

Chris Walton Von innen und von außen. Beethovens Neunte Sinfonie und die $>$ Wagner'sche< Dirigiertradition $\quad 2 \mathrm{I} 8$

Lena-Lisa Wüstendörfer Streit um Fidelio. Gustav Mahler und Felix Weingartner im Disput um Werktreue $\quad 238$

INTERMEZZO

Robert Levin Turning Point to Musical Modernity. Beethoven as Executor of the Legacy of C. P. E. Bach. Concert Lecture 249

INTERPRETATION - INSTRUMENTE, ANALYSE, EDITION

Martin Skamletz «Man hat diese Erweiterung des Tonumfanges seit ein paar Jahren an den Tasteninstrumenten sehr weit getrieben.« Der Umgang mit Grenzen beim späten Mozart und beim frühen Beethoven $\quad 263$ 
Stephan Zirwes Analyse und Interpretation. Adolph Bernhard Marx' Beethoven-Analysen 29I

Michael Ladenburger Was können wir aus Originalhandschriften von Beethoven für eine angemessene Interpretation lernen? 30I

Federica Rovelli Die Skizzenbuch-Ausgaben und ihre mögliche digitale Zukunft 3 I7

Johannes Gebauer Interpretationspraktische Stemmatik. Philologische Methoden in der Interpretationsforschung am Beispiel annotierter Notenausgaben von Rodes 24 Capricen und Beethovens Violinkonzert

John Rink Chopin Copying Chopin 349

Tomasz Herbut Alexander Goldenweiser und Beethovens Sonate op. IIo- eine Spurensuche 366

INTERPRETATION - KREATIVE ANEIGNUNG

Thomas Gartmann Beethoven als sein eigener Interpret. Gedanken zur Bearbeitung der Klaviersonate op. I4/ז für Streichquartett

Ivo Haag Die Sinfonien von Johannes Brahms - (auch) Klaviermusik?

Michael Lehner Das Orchester auf dem Klavier. Welte-Klavierrollen von Gustav Mahler und Richard Strauss als interpretationsanalytische Quellen

Roger Allen “That Is What Music Really Is". Richard Wagner's Reception of Beethoven's Piano Sonata in A Major Op. IOI $43 \mathrm{I}$

Daniel Allenbach Eine heroische< Neunte? Dmitri Schostakowitschs Neunte Sinfonie im Vergleich mit Ludwig van Beethoenvs Sinfonien Nr.3 und 9 44I

Simeon Thompson Beethoven und der Zweite Weltkrieg in der künstlerischen Reflexion der Nachkriegszeit. Stanley Kubricks A Clockwork Orange und Rolf Liebermanns Leonore 40/45 456

Michelle Ziegler Rettungsversuch im Jubiläumsjahr. Mauricio Kagels Aufarbeitung der Beethoven-Rezeption in der Ludwig van-Werkgruppe (I970) 465

Leo Dick Über den späten Beethoven zur >Postidentität،. Die Suche nach liminalen Räumen im gegenwärtigen Musiktheater am Beispiel von Matthias Rebstocks Berliner Produktion Büro für postidentisches Leben $\quad 476$

Elizabeth Waterhouse Choreographic Re-mix. William Forsythe's Trio (I996) and Beethoven's String Quartet No. I5 in a Minor Op. I32 487

László Stachó "Gradus ad Parnassum".

The Purgatory of Instrumental Technique

Namen-, Werk- und Ortsregister 522

Die Autorinnen und Autoren der Beiträge 


\section{Rund um BeEthoven \\ Interpretationsforschung heute • \\ Herausgegeben von Thomas}

Gartmann und Daniel Allenbach 


\section{MUSIKFORSCHUNG DER Hochschule der KÜnste Bern Herausgegeben von Martin Skamletz und Thomas Gartmann Band 14}


0 Dieses Buch ist in gedruckter Form im Dezember 2019 in erster Auflage in der Edition Argus in Schliengen/Markgräflerland erschienen. Gestaltet und gesetzt wurde es im Verlag aus der Seria und der SeriaSans, die von Martin Majoor im Jahre 2000 gezeichnet wurden. Gedruckt wurde es auf Eos, einem holzfreien, säurefreien, chlorfreien und alterungsbeständigen Werkdruckpapier der Papierfabrik Salzer im niederösterreichischen Sankt Pölten. Das Vorsatzpapier Caribic cherry wurde von Igepa in Hamburg geliefert. Rives Tradition, ein Recyclingpapier mit leichter Filznarbung, das für den Bezug des Umschlags verwendet wurde, stellt die Papierfabrik Arjo Wiggins in Issy-les-Moulineaux bei Paris her. Das Kapitalband mit rot-schwarzer Raupe lieferte die Firma Dr. Günther Kast aus Sonthofen im Oberallgäu, die auf technische Gewebe und Spezialfasererzeugnisse spezialisiert ist. Gedruckt und gebunden wurde das Buch von der Firma Bookstation im bayerischen Anzing. Im Internet finden Sie Informationen über das gesamte Verlagsprogramm unter www.editionargus.de, zum Institut Interpretation der Hochschule der Künste Bern unter www.hkb.bfh.ch/interpretation und www.hkb-interpretation.ch. Die Deutsche Nationalbibliothek verzeichnet diese Publikation in der Deutschen Nationalbibliografie; detaillierte bibliografische Daten sind im Internet über www.dnb.de abrufbar. (c) der zeitgleich erschienenen digitalen Version: die Autorinnen und Autoren, 20I9. Dieses Werk ist lizenziert unter einer Creative Commons Namensnennung-Nicht kommerziell 4.0 International Lizenz (CC BY-NC 4.o). DoI: https://doi.org/I0.26045/kp64-6I78 ISBN 978-3-93I264-94-9 